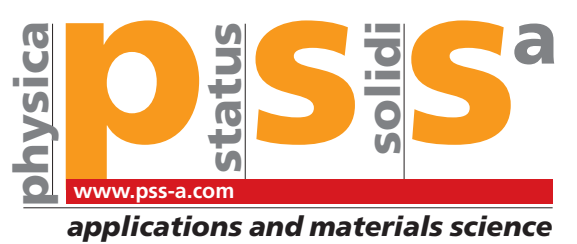

\title{
Metal-oxide-diamond interface investigation by TEM: Toward MOS and Schottky power device behavior
}

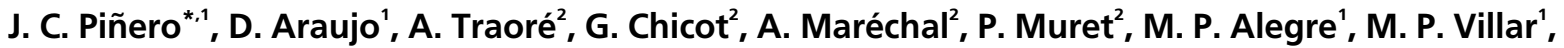 \\ and J. Pernot ${ }^{2}$ \\ ${ }^{1}$ Departamento Ciencia de los Materiales, Universidad de Cádiz, 11510 Puerto Real, Cadiz, Spain \\ ${ }^{2}$ Institut Néel, CNRS-UJF, av. des Martyrs, 38042 Grenoble, France
}

Received 14 March 2014, revised 26 May 2014, accepted 26 May 2014

Published online 23 June 2014

Keywords diamond, oxide characterization, power device, Schottky, TEM

*Corresponding author: e-mail josecarlos.pinero@uca.es, Phone: +34 660584 895, Fax: 956016288

\begin{abstract}
Metal and oxide distribution in diamond metal-oxidesemiconductor (MOS) structures are characterized using several transmission electron microscopy (TEM) modes at nanometric scale. To understand their electrical behavior, oxygen distribution using electron energy loss spectroscopy (EELS) through the layer structure, high-resolution electron
\end{abstract}

microscopy (HREM), and annular dark field (ADF) observations are reported. Oxide thickness variations, as well as oxygen content variations have been identified and characterized at an atomic resolution. The latter allows to understand the related electrical behavior as, for example, leakages or shortcuts.
1 Introduction Diamond is the ultimate semiconductor for high power and high frequency applications due to his high breakdown electric field [1], elevated mobility [2], and the outstanding thermal conductivity. Among such perspectives, diamond-based MOSFET and Schottky diodes are attractive architectures where the conducting or insulating behavior of the device is based on the electrostatic control of the band curvature at the oxide/semiconductor interface.

Previous metal-oxide-semiconductor (MOS) structures performed on diamond exhibited accumulation regime, but no deep depletion or inversion regimes [3-5]. Recent works report investigation of $\mathrm{Al}_{2} \mathrm{O}_{3}$ with hydrogenterminated [6,7] and oxygen-terminated diamond [8]. The latter demonstrates that such MOS capacitors can undergo accumulation, depletion, and deep depletion, but some structures are still presenting some undesired electric behavior.

On the other hand, Schottky diodes, compared to other diamond power devices (bipolar transistor [9], junction field effect transistor [10], Schottky-pn diode [11], etc.) is the most promising because of a very simple structure and a highest breakdown voltage $[1,12]$. However, still architectural progress are required to minimize its serial resistance.
Surface pretreatment and Schottky metal selection are crucial on the development of diamond Schottky power diodes, insofar that is affecting to the barrier heights, the thermal stability and defect density at the interface.

Recently, it has been assumed that the modification of the electrical behavior with thermal treatment of Schottky diodes [13] or with the atomic layer deposition (ALD) $\mathrm{Al}_{2} \mathrm{O}_{3}$ growth conditions in MOS [8] are related with the oxide layer modifications. In particular, leakage currents either in Schottky diodes or in MOSFETs motivates the present nanostructural study of the diamond/oxide interface by scanning transmission microscopy (STEM). Indeed, the electrically measured behavior can be attributed to variations in the oxide thickness as well as differences in the oxygen distribution in the interface. The impact of the oxide layer highlights the importance of a nanostructural characterization of the oxide/diamond interface.

The present contribution analyses the latter interfaces by annular dark field (ADF), conventional transmission electron microscopy (CTEM), high-resolution electron microscopy (HREM), and electron energy loss spectroscopy (EELS) to determine their roughness and sharpness, as well as the oxygen distribution along the oxide layer. 
2 Experimental procedure Samples were prepared using FIB polishing to get electron-transparent specimens with a Quanta 200 3D FIB dual-beam system. Both faces of the specimens were irradiated, at $4^{\circ}$ of incidence, during $45 \mathrm{~s}$ with a $5 \mathrm{kV} / 14 \mathrm{pA}$ beam during the last polish in order to reduce the contribution of the amorphous carbon. The fast diamond tendency to graphitize as well as the effect of the FIB implantation of diamond has been previously studied in literature $[14,15]$.

To evaluate the influence of the oxide variations in power devices, two different structures were analyzed:

(1) Sample A consists in an $\mathrm{Al} / \mathrm{Al}_{2} \mathrm{O}_{3} /$ diamond structure whose electrical behavior was attributed to the possibility of leakages and is corresponding to \#1 in Ref. [8].

(2) Sample B consists in a metallic stack, whose $\mathrm{Zr}$ layer is in contact with an oxygen-terminated low-doped diamond substrate [13].

Samples were observed by CTEM for a first visual characterization of the diamond/oxide interface. Then, EELS profiles and maps were used to identify and characterize the thickness and the relative composition variations of the oxide layers, these profiles were acquired along oxide/diamond interface for an accurate characterization of the thickness variations. Finally, HREM imaging allows characterizing the previously identified oxide layer with atomic resolution. To realize such characterization, a JEOL 2010F transmission electron microscope (with point-to-point resolution of $0.2 \mathrm{~nm}$ ) was used.

The previously discussed procedure allows the nanocharacterization of oxide structures, whose thickness variations may lead to leakages, shortcuts, and undesired electric behavior. Here are studied samples \#A and \#B. Sample \#A is corresponding with \#1 in Ref. [8], an $\mathrm{Al} / \mathrm{Al}_{2} \mathrm{O}_{3} /$ diamond structure consisting in a B-doped diamond layer with a boron concentration of about few $10^{17} \mathrm{~cm}^{-3}$. The deposition was performed at $910^{\circ} \mathrm{C}$ with $\mathrm{CH}_{4} / \mathrm{H}_{2}=2 \%$, $\mathrm{B} / \mathrm{C}=1200 \mathrm{ppm}$ at $830^{\circ} \mathrm{C}$. Diamond surface oxygenation was performed by deep UV ozone treatment. Then, low temperature $\left(100^{\circ} \mathrm{C}\right)$ atomic layer deposition (ALD) was used for the creation of the oxide layer, the precursor used was trimethyllaluminum (TMA), and the oxidant was $\mathrm{H}_{2} \mathrm{O}$. After this, the dielectric was covered by a $100 \mathrm{~nm}$ thick aluminum metal using electron beam evaporator.

On the other hand, sample \#B consists in a metallic stack with a $\mathrm{Zr}$ contact in a boron-doped oxygen-terminated diamond surface, whose electrical behavior has been recently published by Traoré et al. [13]. The boron-doped diamond layer was grown by microwave plasma-enhanced chemical vapor deposition (MPCVD) with $0.75 \%$ of $\mathrm{CH}_{4}$ and $0.5 \%$ of $\mathrm{O}_{2}$ in hydrogen gas, using a 50 Torr pressure and a temperature of $910^{\circ} \mathrm{C}$.

An electron beam evaporator was used to deposit $\mathrm{Zr}$ contacts and the first metallic material was subsequently covered with different cap layers. Tin-doped ITO were deposited to fabricate Schottky contacts. ITO contacts were deposited by sputtering and annealed at $200{ }^{\circ} \mathrm{C}$ during $30 \mathrm{~min}$, allowing crystallization.

$3 \mathrm{Al}_{2} \mathrm{O}_{3}$ for MOS structures $\mathrm{Al}_{2} \mathrm{O}_{3}$ for MOS structures is a wise choice, due to the relative band-gap difference. $\mathrm{Al} / \mathrm{Al}_{2} \mathrm{O}_{3} /$ diamond structure investigations have been recently reported $[6,7]$, particularly, sample \#A was previously studied by Chicot et al. [8], showing field effect in most of the structures. However, some capacitors still presents a peculiar $\mathrm{C}(\mathrm{V})$, that can be attributed to leakages inside the MOS structure. Indeed, the structure developed for such measurement avoid surface leak. Then, imaging techniques are used to reveal the oxide layer configuration. First, ADF (Fig. 1) and CTEM (Fig. 2) observations shows the $\mathrm{Al} / \mathrm{Al}_{2} \mathrm{O}_{3} /$ diamond structure. And second, EELS spectra and profiles (Fig. 3) were acquired in different points of the oxide/diamond interface (corresponding with the numbered positions).

Figure 1 shows an ADF micrography of sample \#A, revealing the presence of $\mathrm{Al}$ grains over the oxide layer. Furthermore, as contrast in ADF imaging is due to the joint contribution of the relative $Z$ number difference and the diffraction effects, $\mathrm{Al}_{2} \mathrm{O}_{3}$ layer appear as a dark contrast. This is not only due to the fact that $\mathrm{Al}_{2} \mathrm{O}_{3}$ is lighter than $\mathrm{Al}$, but is also dependent on diffraction contrast (as can be seen in the $\mathrm{Al}$ layer). The dark contrast of the $\mathrm{Al}_{2} \mathrm{O}_{3}$ is probably largely also due to the amorphous nature of the layer. Additionally, white dashed line in Fig. 1 is used to guide the eyes along the metal-oxide interface, (b)-labeled arrows are used to highlight local oxide thickness variations, finally, (a)-labeled arrows in Fig. 1 shows possible metalsemiconductor contact points.

As can be observed in Fig. 2, oxide's mean thickness is altered in the vicinity of the Al grains. Such behavior can lead to leakages or to shortcuts (if contacting the Al layer with the diamond substrate). Black arrows in Fig. 2 are used to highlight the oxide thickness variations along the

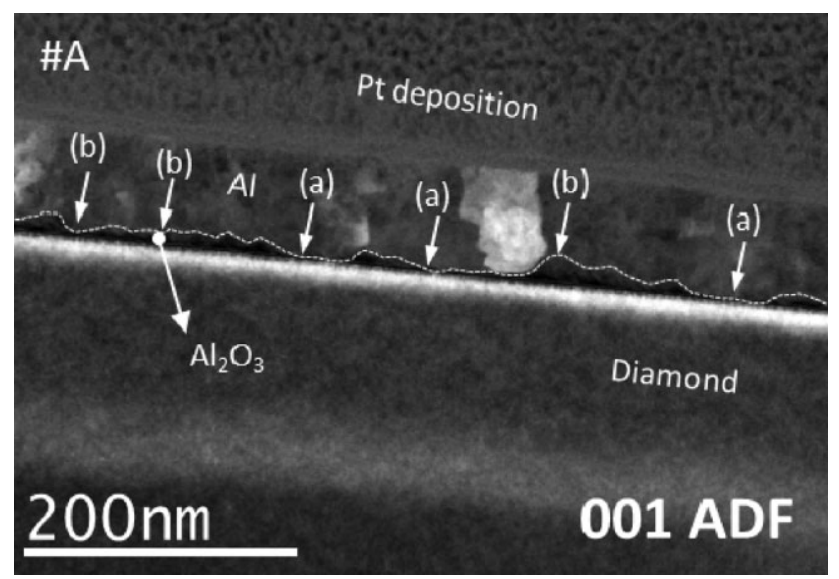

Figure 1 Annular dark field (ADF) micrograph of sample \#A that reveals both, the grain structure along the $\mathrm{Al}$ layer and oxide thickness variations. 


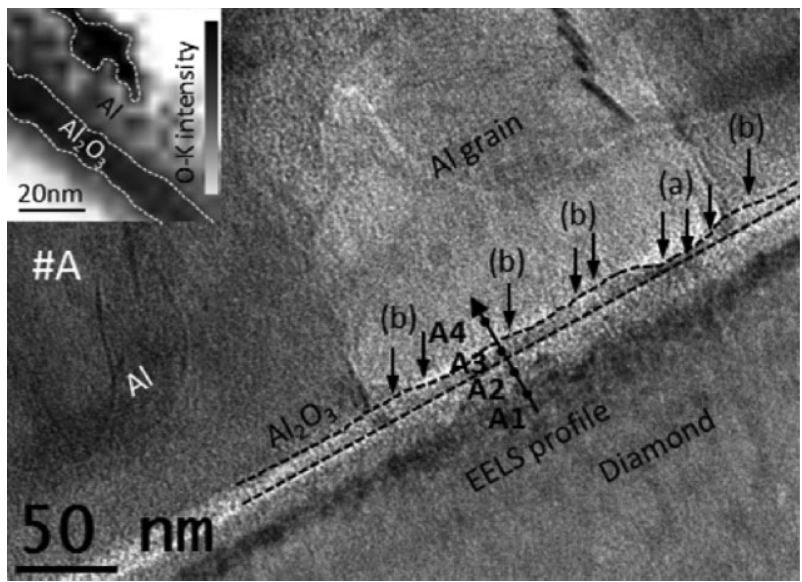

Figure 2 Bright field micrograph of sample \#A, using the 001 reflection. A polycrystalline grain structure is observed in the $\mathrm{Al}$ layer. In the $\mathrm{Al}_{2} \mathrm{O}_{3}$ layer, thickness variations are revealed. Electron energy loss spectroscopy mapping (inset) in another region allows identifying the oxide layer through the observation of the $\mathrm{O}-\mathrm{K}$ peak.

interface. A black dashed line is superimposed to guide the eyes at both diamond/oxide and oxide/Al interfaces.

As the nominal diamond substrate's surface is very smooth, the first interface shows a similar behavior along the structure. In contrary, despite the oxide layer is deposited by low temperature ALD, a relatively rough interface is revealed between oxide/Al.

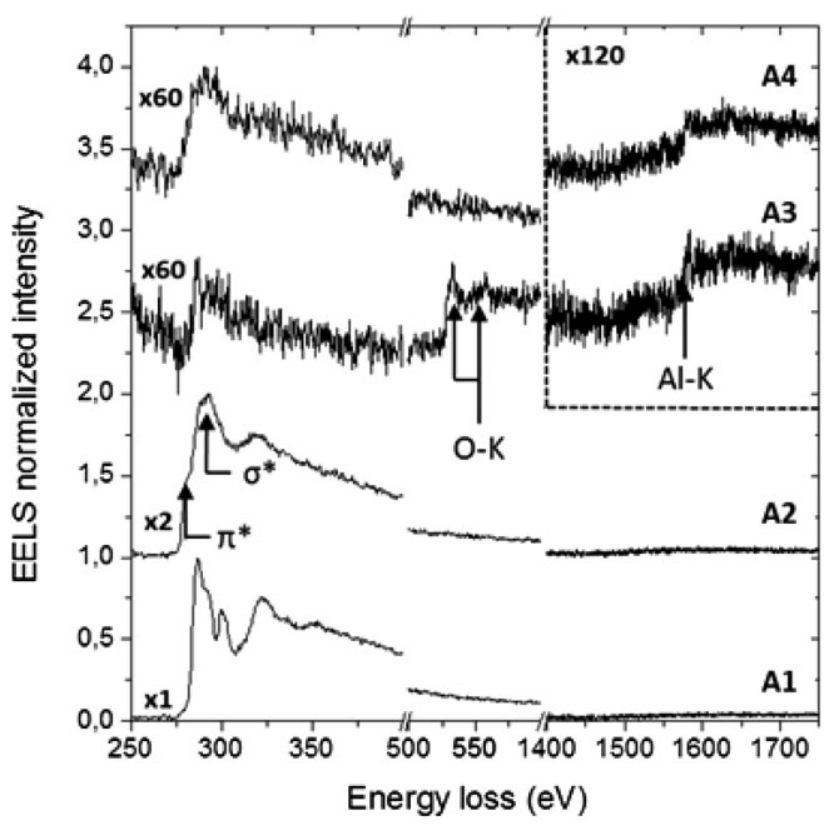

Figure 3 Electron energy loss spectra recorded along the diamond/ oxide structure of \#A (see Fig. 2). $\pi^{*}$ and $\sigma^{*}$ peaks of the carbon $\mathrm{K}$-transition are observed, revealing diamond structure in the substrate (spectrum 1). The $\mathrm{C}$ signal in spectra $\mathrm{A} 2, \mathrm{~A} 3$, and $\mathrm{A} 4$ may be due to $\mathrm{C}$ contamination produced during the FIB operation. The oxygen-K peak transition is observed in spectrum A3.
Variations of the $\mathrm{Al}_{2} \mathrm{O}_{3}$ thickness are shown to have some impact on the further synthesis of the device, insofar that $\mathrm{Al} /$ diamond electric contacts can be produced, leading to shortcuts and undesirable electrical behavior. For example, (b)-labeled arrows in Fig. 2 mark the presence of local maximums of the $\mathrm{Al}_{2} \mathrm{O}_{3}$ thickness corresponding with an $\mathrm{Al}$ grain boundary. Using EELS profiling, a minimum oxide thickness of $4.8 \mathrm{~nm}$ as well as a maximum alumina thickness of $20.4 \mathrm{~nm}$ were measured in the $\mathrm{Al}$ grain/alumina interface. The previously discussed behavior is coherent with the EELS map recorded at $532 \mathrm{eV}$ (color-inverted, oxygen in black) shown in the inset of Fig. 2, which was acquired on a different region of the sample. EELS maps acquired along the interface allow verifying the oxide thickness variations, evidencing also the presence of $\mathrm{Al}_{2} \mathrm{O}_{3}$ islands into the $\mathrm{Al}$ layer, as were shown in inset of Fig. 2.

Figure 3 shows EELS profiles that confirm the presence of the oxide layer. Electron energy loss spectra were acquired in substrate, oxide, and metal layers following the numbered profile of Fig. 2. Spectra were acquired over a $3 \times 3 \mathrm{~nm}^{2}$ area to improve the signal-to-noise relation. It can be observed that the $\mathrm{sp}^{3} \mathrm{C}$-related signal at $285-292 \mathrm{eV}$ is defined only in the diamond substrate, while some lower carbon contamination ( $\mathrm{sp}^{2}$ shape) is observed at $\mathrm{A} 3$ and $\mathrm{A} 4$. $\mathrm{C}$ redeposition during FIB operation and carbon contamination due to long-time EELS data acquisition can also explain the slight presence of the C-related signal in spectra A3 and A4. Spectrum A1 in Fig. 3 shows C-K peak with typical diamond signature, but spectrum A2 in Fig. 3 is presenting graphitization $\left(\pi^{*}\right.$ and $\sigma^{*}$ are revealed near the diamond/ oxide interface). Likewise, the oxygen-K signal showed in spectrum A3 of Fig. 3 is confined on the oxide layer. By using this last result, EELS profiling has been used to explore the oxide layer's homogeneity. A series of profiles were acquired along $1.5 \mu \mathrm{m}$ of the oxide/diamond interface. One of these EELS profiles is shown in Fig. 4, this allows studying the oxygen peak evolution along the oxide layer, revealing an asymmetric oxygen distribution. The relative

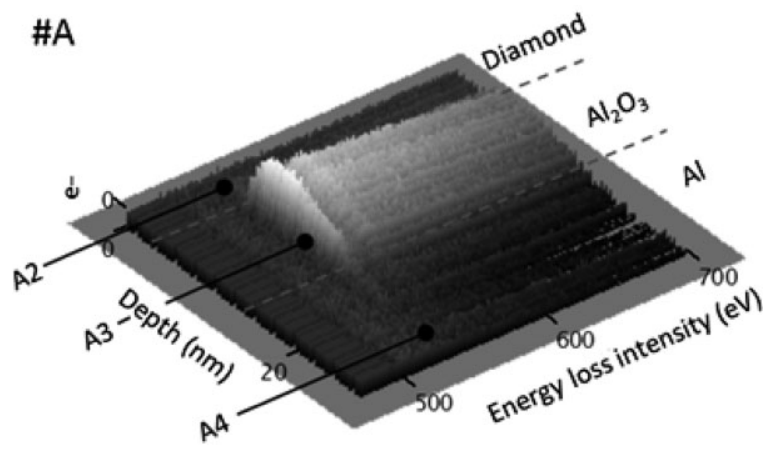

Figure 4 Electron energy loss spectroscopy (EELS) profile in an specific point of the diamond $/ \mathrm{Al}_{2} \mathrm{O}_{3} / \mathrm{Al}$ interface (sample \#A). The $\mathrm{O}-\mathrm{K}$ peak is revealed, the peak intensity along the alumina layer is asymmetrically distributed. The numbered positions are corresponding with the spectra presented in Fig. 3 and with the spatial distribution already shown in Fig. 2. 
intensity of the O-K peak is different depending on the point of the interface, that is an indirect evidence of the oxide thickness inhomogeneity (insofar that the e-beam is passing through a different oxide thickness point by point). These EELS profiles reveal a maximum oxide thickness of $20.4 \mathrm{~nm}$ and a mean thickness of $11.2 \mathrm{~nm}$, as stated above.

EELS profiling and mapping are coherent with CTEM imaging, revealing oxide thickness variations in the $\mathrm{Al}$ grain vicinities. Such behavior could mean a mixture of alumina and aluminum in the metal/oxide interface, and can explain the undesired electrical behavior [8].

$4 \mathrm{Zr}$ for diamond Schottky power diode An outstanding power diode using oxygen-terminated diamond was presented in Ref. [13]. Such device is presenting a high current density and a reverse current that was below the detection threshold. The good electrical behavior of such device was attributed to the possibility of the formation of a thin zirconia film between the oxygen-terminated diamond and the zirconium layer. To verify this assumption, HREM and EELS are here carried out to evidence the presence of oxide in the state of $\mathrm{ZrO}_{2}$ and show its spatial distribution. The latter is evidenced by HREM with EELS certifying the presence of $\mathrm{ZrO}_{2}$.

Figure 5 shows an HREM micrograph of the MOS structure in sample \#B and allows measuring the thickness of the $\mathrm{ZrO}_{2}$ layer with atomic resolution, leading to the detection of an about $4 \AA$ thick oxide layer. Distance between atomic columns of approximately $3 \AA$ can be extracted from an HREM intensity profile. EELS profiling allows identifying the white contrast presented in Fig. 5 as the oxide layer. Squared-dashed line regions in Fig. 5 shows HREM filtered images, revealing [022]-type diamond planes. Fourier transform in the squared regions reveals

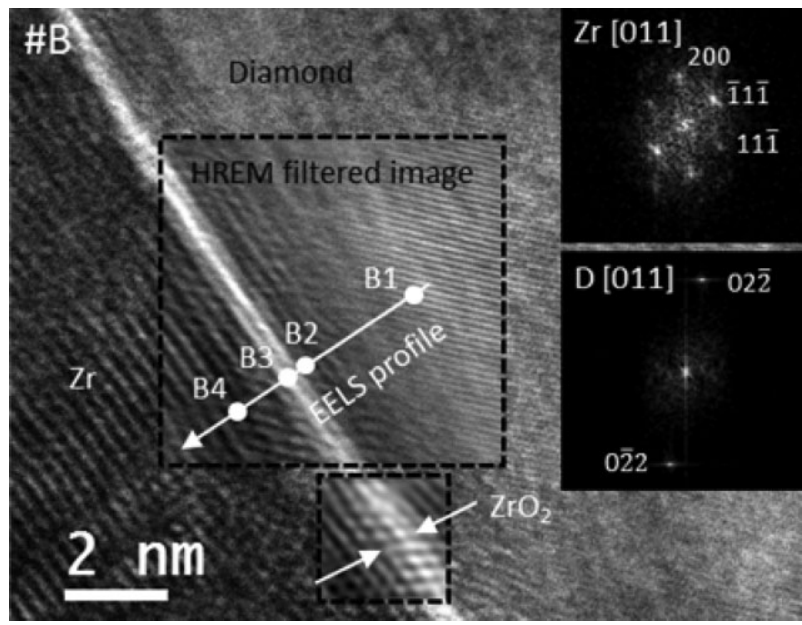

Figure 5 Wiener filtered HREM micrograph of sample \#B. Atomic columns are revealed. Oxide thickness remains constant along the interface. EELS allows identifying a $4 \AA \mathrm{ZrO}_{2}$ layer. Inset shows Fourier transform of the selected filtered area, revealing the [011] pole.
[011] pole. Fourier transform was carried out in the $\mathrm{Zr}$ layer and in the diamond substrate, both are shown as insets in Fig. 5.

By combining the identified [011] pole in the diamond's Fourier transform, the already known diamond's lattice parameter of $3.56 \AA$ and the zirconium's [011]-oriented Fourier transform, a $5 \AA \mathrm{Zr}$ lattice parameter has been calculated. This result is coherent with the lattice parameter of a cubic $\mathrm{Zr}$ structure [16].

On the other hand, EELS spectra were recorded along the white line indicated in Fig. 5, corresponding results are shown in Fig. 6. Spectra B1 and B2 in Fig. 6 have the typical $\mathrm{sp}_{3}$-related shape. The latter shows also a weak $\mathrm{Zr}-\mathrm{M}_{4,5}$ signal due to the spot extension. Presence of $\mathrm{ZrO}_{2}$ layer is demonstrated in spectrum B3 of Fig. 6, with the simultaneous presence of the $\mathrm{O}-\mathrm{K}$ line with the $\mathrm{M}_{4,5}$ and $\mathrm{M}_{3} \mathrm{Zr}$ lines. It also confirms that the white contrast observed in Fig. 5 is, in fact, the zirconia layer.

Using this procedure, three regions are defined in Fig. 5:

(1) Zr layer, confirmed by spectrum B4 in Fig. 6.

(2) Four Angstrom thick $\mathrm{ZrO}_{2}$ layer, identified by EELS (spectrum B3 in Fig. 6).

(3) Diamond substrate.

Thickness of the zirconia layer was homogeneous along the studied interface. This result is in agreement with the assumption of the formation of a thin zirconia layer in the interface of the $\mathrm{Zr}$ deposition with the oxygen-terminated diamond and it can explain the good electric behavior of the associated device [13].

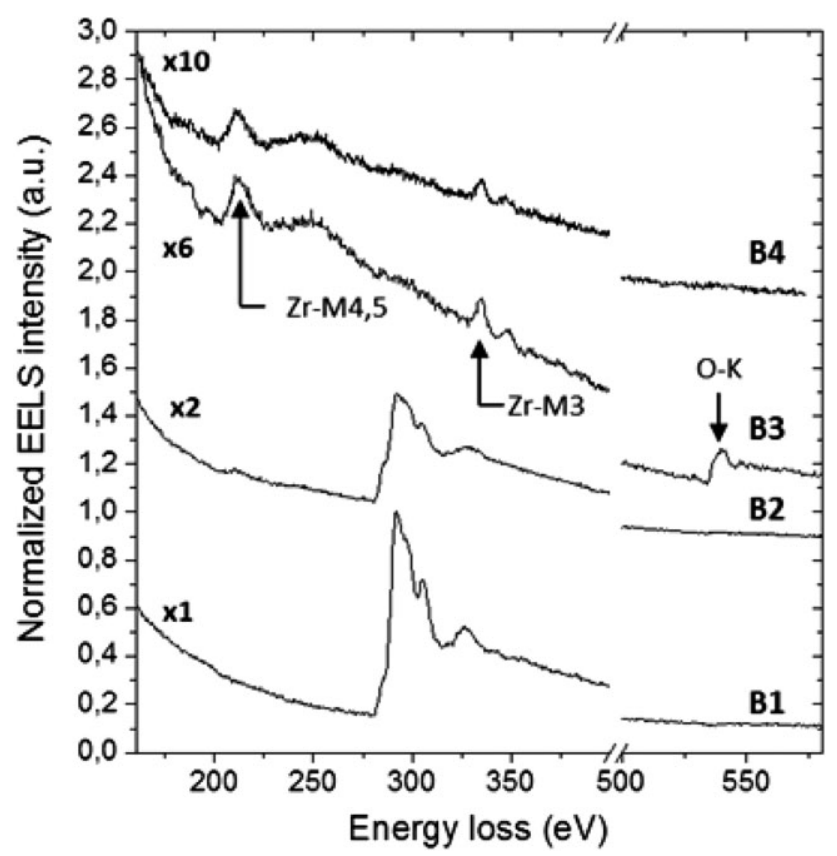

Figure 6 EELS profile of the metal-oxide-semiconductor interface of \#B acquired as shown in Fig. 5. $\mathrm{ZrO}_{2}$ layer is identified. 
5 Conclusions The electrical behavior of the $\mathrm{Al} /$ $\mathrm{Al}_{2} \mathrm{O}_{3}$ /diamond MOS structure (sample \#A), that was previously studied by Chicot el al. [8], has been explained in terms of thickness variations of the oxide layer. Not only the oxide thickness, but also the oxygen distributions inside the oxide layer are varying. This behavior can lead to leakages or to shortcuts ( $\mathrm{Al} /$ diamond contacts) in the associated device.

On the other hand, the electrical behavior of the Schottky diode with a $\mathrm{Zr} / \mathrm{ZrO}_{2}$ /diamond interface (sample \#B), has been explained. The hypothesis of Traoré et al. [13] about the formation of a $4 \AA$ thick zirconia layer has been demonstrated. The stability and homogeneity of such layer along the metal/oxide interface remains constant, explaining the electrically measured behavior.

Variations in the oxygen distribution, oxide/metal mixtures and oxide thickness variations have been characterized. These alterations can lead to undesired electric behavior in the associated electric device. Finally, oxide thickness variations have been identified with atomic resolution.

Acknowledgements The authors thank "Ministerio de Ciencia e Innovación" for the grants BES-2010-039524 and EEBB-I-13-07696 of the project TEC2009-11399.

\section{References}

[1] T. P. Chow, W. Huang, J. Yang, and J. E. Butler, Int. J. High Speed Electron. Syst. 14, 872 (2004).
[2] J. Hammersberg, J. Isberg, E. Johansson, T. Wikstrom, D. J. Twitchen, A. J. Whitehead, S. E. Coe, and G. A. Scarsbrook, Science 297, 1670 (2002).

[3] M. Imura, R. Hayakawa, E. Watanabe, M. Y. Liao, Y. Koide, and H. Amano, Phys. Status Solidi RRL 5(3), 125 (2011).

[4] H. Sato, M. Kasu, and K. Hirama, Appl. Phys. Lett. 5, 025701 (2012).

[5] Y. Yokota, N. Kawakami, T. Tachibana, K. Hayashi, and K. Kobashi, Diamond Relat. Mater. 14, 2015 (2005).

[6] A. Daicho, A. Hirawira, S. Kurihara, Y. Yokoyama, and H. Kawarada, J. Appl. Phys. 112, 124504 (2012).

[7] M. Y. Liao, J. W. Liu, M. Imura, H. Li, and Y. Koide, Appl. Phys. Lett. 101, 232907 (2012).

[8] G. Chicot, A. Marechal, R. Motte, P. Muret, E. Gheeraert, and J. Pernot, Appl. Phys. Lett. 102, 242108 (2013).

[9] K. Oyama, H. Kato, T. Makino, M. Ogura, D. Takeuchi, and S. Yamasaki, Diamond Relat. Mater. 27-28, 19 (2012).

[10] Y. Hoshino, T. Iwazaki, K. Tsuzuki, H. Kato, T. Makino, M. Ogura, D. Taekuchi, T. Matsumoto, H. Okushi, S. Yamasaki, and M. Hatano, Appl. Phys. Express 5, 091301 (2012).

[11] S. Tanimoto, T. Makino, Y. Hayashi, H. Kato, N. Tokuda, M. Ogura, D. Takeuchi, K. Oyama, H. Ohashi, H. Okushi, and S. Yamasaki, Appl. Phys. Lett. 94, 262101 (2009).

[12] P. Muret, P. N. Volpe, J. Pernot, F. Omnès, T. Teraji, F. Jomard, D. Planson, P. Brosselard, N. Dheilly, B. Vergne, and S. Scharnholtz, Appl. Phys. Lett. 97, 223501 (2010).

[13] A. Traoré, P. Muret, A. Fiori, D. Eon, E. Gheeraert, and J. Pernot, Appl. Phys. Lett. 104, 052105 (2014).

[14] W. R. McKenzie, M. Z. Quadir, M. H. Gass, and P. R. Munroe, Diamond Relat. Mater. 20, 1125 (2011).

[15] Y. Q. Chen, F. Z. Tang, and L. C. Zhang, J. Mater. Sci. Nanotechnol. 531-532, 592 (2013).

[16] R. A. Ploc, J. Nucl. Mater. 99, 124 (1981). 